\title{
Effect of Varying StepSizes in Numerical Approximation of Stochastic Differential Equations Using One Step Milstein Method
}

\author{
Sunday Jacob Kayode ${ }^{1}$, Akeem Adebayo Ganiyu ${ }^{2, *}$ \\ ${ }^{1}$ Department of Mathematical Sciences, Federal University of Technology, Akure, Nigeria \\ ${ }^{2}$ Mathematics Department, Adeyemi College of Education, Ondo, Nigeria \\ Email address: \\ cosjkay57@yahoo.com (S. J. Kayode), ganiyuiwajowa@gmail.com (A. A. Ganiyu)
}

\section{To cite this article:}

Sunday Jacob Kayode, Akeem Adebayo Ganiyu. Effect of Varying StepSizes in Numerical Approximation of Stochastic Differential Equations Using One Step Milstein Method. Applied and Computational Mathematic. Vol. 4, No. 5, 2015, pp. 351-362.

doi: $10.11648 /$ j.acm. 20150405.14

\begin{abstract}
This paper examines the effect of varying stepsizes in finding the approximate solution of stochastic differential equations (SDEs). One step Milstein method (MLSTM) for solution of general first order stochastic differential equations (SDEs) has been derived using Itô Lemma and Euler-Maruyama Method as supporting tools. Two problems in the form of first order SDEs have been considered. The method of solution used is one step Milstein method. The absolute errors were calculated using the exact solution and numerical solution. Comparison of varying the stepsizes was achieved using mean absolute error criterion. The results showed that the mean absolute error due to approximation decreases as the stepsizes decreases. The order of convergence is approximately 1, which indicates the accuracy of the method. Also, the effect of varying stepsizes can also be identified using graphical method constructed for various stepsizes.
\end{abstract}

Keywords: Stochastic Differential Equations, Itô Lemma, Euler-Maruyama Method, Milstein Method, Wiener Process, Wiener Increment, Black Scholes Option Price Model, StepSizes

\section{Introduction}

Consider a stochastic differential equation of the form

$$
d X=f(t, X(t)) d t+g(t, X(t)) d W(t), X\left(t_{0}\right)=X_{0}
$$

where $f:[0, T] \times \mathbb{R}^{n} \rightarrow \mathbb{R}^{n}, g:[0, T] \times \mathbb{R}^{n} \rightarrow \mathbb{R}^{n \times m}$ are the drift function and diffusion function respectively. $W(t)$ is Wiener process.

Many researchers have worked on SDE of the form (1), among these are Platen (1992) who worked on introductory aspects of SDEs, Higham (2001) who worked on an algorithmic introduction to numerical simulation of SDEs, Burrage et al (2000) who worked on numerical solution of SDEs and discusses stability issues, Burrage (2004) who worked on the overview of numerical methods for strong solution of SDEs, Anna (2010) who worked on economic Runge-Kutta methods with weak second order for SDEs, Razaeyan and Farnoosh (2010) who worked on analytical solution of SDEs with application to Kalman-Bucky filter in modeling RC circuit, Fadugba et al (2013) who worked on convergence of Euler-Maruyama and Milstein scheme for solution of stochastic ordinary differential equations, Bokor (2003) who worked on stochastic stable one step approximations of solution of SDEs, Sauer (2013) who worked on computational solution of SDEs and Akinbo et al (2015) who worked on numerical solution of stochastic differential equations.

The objective of this paper is to develop one step Milstein method for solution of SDE (1) and apply it to solve two problems in the form of first order SDEs. Absolute errors will be determined at various point in the interval $[0, T]$, where $T=1$. The absolute errors will be determined by finding the absolute value of the difference between the exact solution and numerical solution of (1) using one-step Milstein method. Obtaining these values for various values of $t \in[0, T]$ will allow us to obtain mean absolute error. The order of convergence of the method will be determined from the stepsizes used and the mean absolute error obtained. Fadugba et al (2013) consider the effect of using single step 
size for solution of SDE (1).

Integrating equation (1) from 0 to $t$, we have the following integral.

$$
X(t)=X_{o}+\int_{o}^{t} f(s, X(s)) d s+\int_{o}^{t} g(s, X(s)) d W(s)
$$

The first integral at the right hand side of equation (2) is called Riemman integral while the second integral is called Itô or stochastic integral.

\section{Research Methodology}

To determine the solution of $\operatorname{SDE}(1)$, we shall use one step Milstein method (MLSTM). This method was used by Higham (2001) by considering an autonomous system of stochastic differential equations. Here, we shall consider the derivation of one step MLSTM for the solution of general first order stochastic differential equations (SDEs) of the form (1). For this derivation, the following Lemma shall be a vital tool.

Consider a SDE of equation (1).

Also, consider a function $U=U(X)$. Applying Taylor's theorem to $U$, we have

$$
d U=\frac{d U}{d X} \frac{(d X)^{1}}{1 !}+\frac{d^{2} U}{d X^{2}} \frac{(d X)^{2}}{2 !}+\ldots
$$

Substituting (1) into (3), gives

$$
\begin{gathered}
d U=\frac{d U}{d X}\{f(t, X(t)) d t+g(t, X(t)) d W(t)\}+\frac{1}{2} \frac{d^{2} U}{d X^{2}}\left\{(f(t, X(t)))^{2}(d t)^{2}+2 f(t, X(t)) g(t, X(t)) d t d W(t)\right. \\
\left.+(g(t, X(t)))^{2}(d W(t))^{2}\right\}+\ldots
\end{gathered}
$$

Here, we can assume that $U=U(t, X(t))$. Also, we can assume as in Oksendal (1998) that as $d t \rightarrow 0$, then $(d W(t))^{2} \rightarrow E(d W(t))^{2}=d t$. Also, as $d t^{2} \rightarrow 0, d t d W(t) \rightarrow 0$.

Equation (4) then becomes

$$
d U=\left(f(t, X(t)) \frac{d U}{d X}+\frac{1}{2}(g(t, X(t)))^{2} \frac{d^{2} U}{d X^{2}}\right) d t+g(t, X(t)) \frac{d U}{d X} d W(t)
$$

Equation (5) is called Itô Lemma (or Stochastic Chain Rule) obtained from (Stochastic-Taylor series expansion), that is Taylor series expansion of SDE (1).

\section{Derivation of One Step Milstein Method (MLSTM)}

In this paper, forward difference approach shall be considered instead of backward difference methods of
Higham (2001) and Richardson (2009). MLSTM will be derived from Itô Lemma (5) by using discritised interval $[0, T]$ as $0<\tau_{0}<\tau_{1}<\cdots<\tau_{j}<\tau_{j+1}=T$. Let $\delta t=\frac{T}{L}$ be the stepsize defined as $\delta t:=\tau_{j+1}-\tau_{j}$, where $L$ are some integer and $\tau_{j}=j \delta t$. In the integral of equation (5), if it is assumed that $t=s$ and $t=\tau_{j}$, the equation becomes

$$
\begin{aligned}
\int_{\tau_{j}}^{s} d U(\tau, X(\tau))=\int_{\tau_{j}}^{s}\left\{f(\tau, X(\tau)) \frac{d U(\tau, X(\tau))}{d X}+\frac{1}{2}(g(\tau, X(\tau)))^{2} \frac{d^{2} U(\tau, X(\tau))}{d X^{2}}\right\} d \tau+\int_{\tau_{j}}^{s}\left\{g(\tau, X(\tau)) \frac{d U(\tau, X(\tau))}{d X}\right\} d W(\tau) \\
\Rightarrow U(s, X(s))=U\left(\tau_{j}, X\left(\tau_{j}\right)\right)+\int_{\tau_{j}}^{s}\left\{f(\tau, X(\tau)) \frac{d U(\tau, X(\tau))}{d X}+\frac{1}{2}(g(\tau, X(\tau)))^{2} \frac{d^{2} U(\tau, X(\tau))}{d X^{2}}\right\} d \tau \\
+\int_{\tau_{j}}^{s}\left\{g(\tau, X(\tau)) \frac{d U(\tau, X(\tau))}{d X}\right\} d W(\tau) \\
\Rightarrow U(s, X(s))=U\left(\tau_{j}, X\left(\tau_{j}\right)\right)+\int_{\tau_{j}}^{s}\left\{f(\tau, X(\tau)) \frac{d}{d X}+\frac{1}{2}(g(\tau, X(\tau)))^{2} \frac{d^{2}}{d X^{2}}\right\} U(\tau, X(\tau)) d \tau \\
+\int_{\tau_{j}}^{s}\left\{g(\tau, X(\tau)) \frac{d}{d X}\right\} U(\tau, X(\tau)) d W(\tau)
\end{aligned}
$$


Assume that

$$
D^{0}=f(\tau, X(\tau)) \frac{d}{d X}+\frac{1}{2}(g(\tau, X(\tau)))^{2} \frac{d^{2}}{d X^{2}} \text { and } D^{1}=g(\tau, X(\tau)) \frac{d}{d X}
$$

Equation (8) becomes

$$
U(s, X(s))=U\left(\tau_{j}, X\left(\tau_{j}\right)\right)+\int_{\tau_{j}}^{s} D^{0} U(\tau, X(\tau)) d \tau+\int_{\tau_{j}}^{s} D^{1} U(\tau, X(\tau)) d W(\tau)
$$

Let $U=f$ and $U=g$ in (10), this respectively gives

$$
\begin{gathered}
f(s, X(s))=f\left(\tau_{j}, X\left(\tau_{j}\right)\right)+\int_{\tau_{j}}^{s} D^{0} f(\tau, X(\tau)) d \tau+\int_{\tau_{j}}^{s} D^{1} f(\tau, X(\tau)) d W(\tau) \\
g(s, X(s))=g\left(\tau_{j}, X\left(\tau_{j}\right)\right)+\int_{\tau_{j}}^{s} D^{0} g(\tau, X(\tau)) d \tau+\int_{\tau_{j}}^{s} D^{1} g(\tau, X(\tau)) d W(\tau)
\end{gathered}
$$

Given the integral form of the one-step Euler-Maruyama method of the form

$$
X\left(\tau_{j+1}\right)=X\left(\tau_{j}\right)+\int_{\tau_{j}}^{\tau_{j+1}} f(s, X(s)) d s+\int_{\tau_{j}}^{\tau_{j+1}} g(s, X(s)) d W(s)
$$

Applying equations (11) and (12) to the integral terms involving $\tau_{i}$ at the lower limit of the integrals in equation (13), gives

$$
\begin{gathered}
X\left(\tau_{j+1}\right)=X\left(\tau_{j}\right)+\int_{\tau_{j}}^{\tau_{j+1}}\left\{f\left(\tau_{j}, X\left(\tau_{j}\right)\right)+\right. \\
\left.\int_{\tau_{j}}^{s} D^{0} f(\tau, X(\tau)) d \tau+\int_{\tau_{j}}^{s} D^{1} f(\tau, X(\tau)) d W(\tau)\right\} d s+\int_{\tau_{j}}^{\tau_{j+1}}\left\{g\left(\tau_{j}, X\left(\tau_{j}\right)\right)\right. \\
\left.+\int_{\tau_{j}}^{s} D^{0} g(\tau, X(\tau)) d \tau+\int_{\tau_{j}}^{s} D^{1} g(\tau, X(\tau)) d W(\tau)\right\} d W(s)
\end{gathered}
$$

Expanding and rearranging, we have

$$
\begin{aligned}
X\left(\tau_{j+1}\right) & =X\left(\tau_{j}\right)+\int_{\tau_{j}}^{\tau_{j+1}} f\left(\tau_{j}, X\left(\tau_{j}\right)\right) d s+\int_{\tau_{j}}^{\tau_{j+1}} g\left(\tau_{j}, X\left(\tau_{j}\right)\right) d W(s)+\int_{\tau_{j}}^{\tau_{j+1}} \int_{\tau_{j}}^{s} D^{1} g(\tau, X(\tau)) d W(\tau) d W(s) \\
& +\int_{\tau_{j}}^{\tau_{j+1}} \int_{\tau_{j}}^{s} D^{0} f(\tau, X(\tau)) d \tau d s+\int_{\tau_{j}}^{\tau_{j+1}} \int_{\tau_{j}}^{s} D^{1} f(\tau, X(\tau)) d W(\tau) d s+\int_{\tau_{j}}^{\tau_{j+1}} \int_{\tau_{j}}^{s} D^{0} g(\tau, X(\tau)) d \tau d W(s)
\end{aligned}
$$

Using the conventional quadrature rule, equation (15) can be written as

$$
\begin{aligned}
X\left(\tau_{j+1}\right)= & X\left(\tau_{j}\right)+\left(\tau_{j+1}-\tau_{j}\right) f\left(\tau_{j}, X\left(\tau_{j}\right)\right)+g\left(\tau_{j}, X_{j}\right)\left(W\left(\tau_{j+1}\right)-W\left(\tau_{j}\right)\right)+\int_{\tau_{j}}^{\tau_{j+1}} \int_{\tau_{j}}^{s} D^{1} g(\tau, X(\tau)) d W(\tau) d W(s) \\
& +\int_{\tau_{j}}^{\tau_{j+1}} \int_{\tau_{j}}^{s} D^{0} f(\tau, X(\tau)) d \tau d s+\int_{\tau_{j}}^{\tau_{j+1}} \int_{\tau_{j}}^{s} D^{1} f(\tau, X(\tau)) d W(\tau) d s+\int_{\tau_{j}}^{\tau_{j+1}} \int_{\tau_{j}}^{s} D^{0} g(\tau, X(\tau)) d \tau d W(s)
\end{aligned}
$$

The first three terms at the right hand side of equation (16) is one-step Euler-Maruyama method. To obtain one-step Milstein Method, we expand $D^{1} g(\tau, X(\tau))$ term using the definition of the operator $D^{1}$ of equation (9) and the formula for $g(s, X(s))$ of equation (12). This gives

$$
D^{1} g(\tau, X(\tau))=D^{1} g\left(\tau_{j}, X\left(\tau_{j}\right)\right)+\int_{\tau_{j}}^{s} D^{0} D^{1} g(n, X(n)) d n+\int_{\tau_{j}}^{s} D^{1} D^{1} g(n, X(n)) d W(n)
$$

for integral terms involving $\tau_{j+r}, r=0$ at the lower limit of the integral (16).

Using (17) in (16) gives

$$
\begin{gathered}
X\left(\tau_{j+1}\right)=X\left(\tau_{j}\right)+\left(\tau_{j+1}-\tau_{j}\right) f\left(\tau_{j}, X\left(\tau_{j}\right)\right)+g\left(\tau_{j}, X_{j}\right)\left(W\left(\tau_{j+1}\right)-W\left(\tau_{j}\right)\right) \\
+\int_{\tau_{j}}^{\tau_{j+1}} \int_{\tau_{j}}^{s}\left\{D^{1} g\left(\tau_{j}, X\left(\tau_{j}\right)\right)+\int_{\tau_{j}}^{s} D^{0} D^{1} g(n, X(n)) d n+\int_{\tau_{j}}^{s} D^{1} D^{1} g(n, X(n)) d W(n)\right\} d W(\tau) d W(s)
\end{gathered}
$$




$$
+\int_{\tau_{j}}^{\tau_{j+1}} \int_{\tau_{j}}^{s} D^{0} f(\tau, X(\tau)) d \tau d s+\int_{\tau_{j}}^{\tau_{j+1}} \int_{\tau_{j}}^{s} D^{1} f(\tau, X(\tau)) d W(\tau) d s+\int_{\tau_{j}}^{\tau_{j+1}} \int_{\tau_{j}}^{s} D^{0} g(\tau, X(\tau)) d \tau d W(s)
$$

Expansion of (18) gives

$$
\begin{aligned}
X\left(\tau_{j+1}\right)= & X\left(\tau_{j}\right)+\left(\tau_{j+1}-\tau_{j}\right) f\left(\tau_{j}, X\left(\tau_{j}\right)\right)+g\left(\tau_{j}, X_{j}\right)\left(W\left(\tau_{j+1}\right)-W\left(\tau_{j}\right)\right)+\int_{\tau_{j}}^{\tau_{j+1}} \int_{\tau_{j}}^{s} D^{1} g\left(\tau_{j}, X\left(\tau_{j}\right)\right) d W(\tau) d W(s) \\
& +\int_{\tau_{j}}^{\tau_{j+1}} \int_{\tau_{j}}^{s} \int_{\tau_{j}}^{s} D^{0} D^{1} g(n, X(n)) d n d W(\tau) d W(s)+\int_{\tau_{j}}^{\tau_{j+1}} \int_{\tau_{j}}^{s} \int_{\tau_{j}}^{s} D^{1} D^{1} g(n, X(n)) d W(n) d W(\tau) d W(s) \\
& +\int_{\tau_{j}}^{\tau_{j+1}} \int_{\tau_{j}}^{s} D^{0} f(\tau, X(\tau)) d \tau d s+\int_{\tau_{j}}^{\tau_{j+1}} \int_{\tau_{j}}^{s} D^{1} f(\tau, X(\tau)) d W(\tau) d s+\int_{\tau_{j}}^{\tau_{j+1}} \int_{\tau_{j}}^{s} D^{0} g(\tau, X(\tau)) d \tau d W(s)
\end{aligned}
$$

By definition $D^{1}=g(\tau, X(\tau)) \frac{d}{d X}$ c.f.(9)

Using (9) in equation (19), this can be written as

$$
\begin{gathered}
X\left(\tau_{j+1}\right)=X\left(\tau_{j}\right)+\left(\tau_{j+1}-\tau_{j}\right) f\left(\tau_{j}, X\left(\tau_{j}\right)\right)+g\left(\tau_{j}, X_{j}\right)\left(W\left(\tau_{j+1}\right)-W\left(\tau_{j}\right)\right) \\
+g\left(\tau_{j}, X\left(\tau_{j}\right)\right) g^{\prime}\left(\tau_{j}, X\left(\tau_{j}\right)\right) \int_{\tau_{j}}^{\tau_{j+1}} \int_{\tau_{j}}^{s} d W(\tau) d W(s)+\int_{\tau_{j}}^{\tau_{j+1}} \int_{\tau_{j}}^{s} \int_{\tau_{j}}^{s} D^{0} D^{1} g(n, X(n)) d n d W(\tau) d W(s) \\
+\int_{\tau_{j}}^{\tau_{j+1}} \int_{\tau_{j}}^{s} \int_{\tau_{j}}^{s} D^{1} D^{1} g(n, X(n)) d W(n) d W(\tau) d W(s)+\int_{\tau_{j}}^{\tau_{j+1}} \int_{\tau_{j}}^{s} D^{0} f(\tau, X(\tau)) d \tau d s \\
+\int_{\tau_{j}}^{\tau_{j+1}} \int_{\tau_{j}}^{s} D^{1} f(\tau, X(\tau)) d W(\tau) d s+\int_{\tau_{j}}^{\tau_{j+1}} \int_{\tau_{j}}^{s} D^{0} g(\tau, X(\tau)) d \tau d W(s)
\end{gathered}
$$

Where, $g^{\prime}\left(\tau_{j}, X\left(\tau_{j}\right)\right)=\frac{d}{d X} g\left(\tau_{j}, X\left(\tau_{j}\right)\right)$.

The one-step MLSTM follow from the first four terms at the right hand side of equation (20). The method is

$$
\begin{aligned}
X\left(\tau_{j+1}\right) & =X\left(\tau_{j}\right)+\left(\tau_{j+1}-\tau_{j}\right) f\left(\tau_{j}, X\left(\tau_{j}\right)\right)+g\left(\tau_{j}, X_{j}\right)\left(W\left(\tau_{j+1}\right)-W\left(\tau_{j}\right)\right) \\
& +g\left(\tau_{j}, X\left(\tau_{j}\right)\right) g^{\prime}\left(\tau_{j}, X\left(\tau_{j}\right)\right) \int_{\tau_{j}}^{\tau_{j+1}} \int_{\tau_{j}}^{s} d W(\tau) d W(s)
\end{aligned}
$$

Having evaluated the double integral at the right hand side of equation (21) using Itô formula, we have

$$
\begin{aligned}
X\left(\tau_{j+1}\right)= & X\left(\tau_{j}\right)+\left(\tau_{j+1}-\tau_{j}\right) f\left(\tau_{j}, X\left(\tau_{j}\right)\right)+g\left(\tau_{j}, X_{j}\right)\left(W\left(\tau_{j+1}\right)-W\left(\tau_{j}\right)\right) \\
& +\frac{1}{2} g\left(\tau_{j}, X\left(\tau_{j}\right)\right) g^{\prime}\left(\tau_{j}, X\left(\tau_{j}\right)\right)\left(\left(W\left(\tau_{j+1}\right)-W\left(\tau_{j}\right)\right)^{2}-\left(\tau_{j+1}-\tau_{j}\right)\right)
\end{aligned}
$$

Define $X\left(\tau_{j+r}\right):=X_{j+r}, r=0,1 ; \delta W_{j}:=\left(W\left(\tau_{j+r}\right)-W\left(\tau_{j+r-1}\right)\right), r=1$ and $\delta t:=\left(\tau_{j+r}-\tau_{j+r-1}\right), r=1$.

Equation(22) can now be written as

$$
\begin{gathered}
X_{j+1}=X_{j}+\delta t f\left(\tau_{j}, X_{j}\right)+g\left(\tau_{j}, X_{j}\right) \delta W_{j}+\frac{1}{2} g\left(\tau_{j}, X_{j}\right) g^{\prime}\left(\tau_{j}, X_{j}\right)\left(\left(W\left(\tau_{j+1}\right)-W\left(\tau_{j}\right)\right)^{2}-\left(\tau_{j+1}-\tau_{j}\right)\right), j=0,1,2, \cdots, L . \\
X_{j+1}=X_{j}+f\left(\tau_{j}, X_{j}\right) \delta t+g\left(\tau_{j}, X_{j}\right) \delta W_{j}+\frac{1}{2} g\left(\tau_{j}, X_{j}\right) g^{\prime}\left(\tau_{j}, X_{j}\right)\left(\left(d W_{j}\right)^{2}-\delta t\right), j=0,1,2, \cdots, L
\end{gathered}
$$

The method in equation (23) was considered by Higham (2001) using backward difference. In this paper, we shall apply this method to the SDE (1) using discritised interval $[0, T]$ as $0<\tau_{0}<\tau_{1}<\cdots<\tau_{j}<\tau_{j+1}=T$. Let $\delta t=\frac{T}{L}$ be the stepsize defined as $\delta t:=\tau_{j+1}-\tau_{j}$, where $N$ are some integer and $\tau_{j}=j \delta t$. The $\delta t$-space path increment $d W_{j}:=W_{j}-W_{j-1}$ will be approximated by summing the underlying $d t$-space increments as established by Higham (2001) using

$$
W i n c=\operatorname{sum}\left(d W\left(R^{*}(j-1)+1: R^{*} j\right)\right.
$$

Wiener increment $d W$ will be generated in MATLAB 
over the space intervals by using $d W:=\operatorname{sqrt}(d t) * \operatorname{rand}(1, N)$. For computational purpose, we shall assume that $\mathrm{R}=1, \mathrm{Dt}=\mathrm{R} * \mathrm{dt}$ and $\mathrm{L}=\mathrm{N} / \mathrm{R}$.

We can now examine the effect of varying step sizes in numerical approximation of stochastic differential Equations using one step Milstein Method.

\section{Effect of Varying StepSizes in Numerical Approximation of Stochastic Differential Equations Using One Step Milstein Method.}

In this section, we will consider two problems in the form of first order stochastic differential equation (1) to investigate the effect of varying step sizes when finding the solution of SDEs using one step MLSTM of equation (24).
Problem 1

$$
d X(t)=\mu X(t) d t+\sigma X(t) d W(t), X\left(t_{0}\right)=X_{0}
$$

where $\mu=0.0002$ and $\sigma=0.0001$ are arbitrary values.

The exact solution of the SDE (26) is

$$
X(t)=X_{0} \exp \left(\left(\mu-0.5 \sigma^{2}\right) t+\sigma W(t)\right)
$$

Problem 1 is the Black-Scholes option price model with a drift $\mu$ and diffusion coefficient $\sigma$. The problem was also used by Higham (2001) and Sauer (2013).

The following stepsizes shall be used to carry out the investigation, $2^{-4}, 2^{-5}, 2^{-6}, 2^{-7}, 2^{-8}, 2^{-9}$. We will assume here that $X(0)=1$.

Table 1. Results of problem 1 showing the effect of using step size $2^{-4}$.

\begin{tabular}{llll}
\hline t-value & Exact Solution & Numerical Solution & Absolute Error \\
\hline 0.062500 & 1.000035213412162 & 1.000035213050127 & $3.62035069 \mathrm{e}-10$ \\
0.125000 & 0.999992194240297 & 0.9999921944944152 & $2.53855270 \mathrm{e}-10$ \\
0.187500 & 0.999998717493128 & 0.999998717743567 & $2.50439003 \mathrm{e}-10$ \\
0.250000 & 1.000012935644641 & 1.000012935795483 & $1.50841784 \mathrm{e}-10$ \\
0.312500 & 0.999974930990167 & 0.999974931694190 & $7.04022729 \mathrm{e}-10$ \\
0.375000 & 0.999978328502518 & 0.999978329242195 & $7.39676542 \mathrm{e}-10$ \\
0.437500 & 0.999988796767910 & 0.999988797454864 & $6.86954160 \mathrm{e}-10$ \\
0.500000 & 0.999951804297058 & 0.999951805524518 & $1.22746024 \mathrm{e}-09$ \\
0.562500 & 0.999984007130832 & 0.999984008033925 & $9.03092601 \mathrm{e}-10$ \\
0.625000 & 1.000014922754158 & 1.000014923348964 & $5.94805760 \mathrm{e}-10$ \\
\hline
\end{tabular}

The mean absolute error is $5.873183162030670 \mathrm{e}-010$

Table 1 above shows the exact solution, numerical solution and absolute error for the effect of using stepsize $2^{-4}$. (See Appendix 1 for the graph of the results on Table 1).

Table 2. Results of problem 1 showing the effect of using stepsize $2^{-5}$

\begin{tabular}{llll}
\hline t-value & Exact Solution & Numerical Solution & Absolute Error \\
\hline 0.062500 & 0.999989302989965 & 0.999989303095894 & $1.05928821 \mathrm{e}-10$ \\
0.125000 & 0.999998791743439 & 0.999998791829126 & $8.56869020 \mathrm{e}-11$ \\
0.187500 & 0.999969143626926 & 0.999969143936974 & $3.10047432 \mathrm{e}-10$ \\
0.250000 & 0.999945211010976 & 0.999945211509657 & $4.98681429 \mathrm{e}-10$ \\
0.312500 & 0.999984664319081 & 0.999984664610261 & $2.91180635 \mathrm{e}-10$ \\
0.375000 & 1.000048162051462 & 1.000048161984859 & $6.66029454 \mathrm{e}-11$ \\
0.437500 & 1.000031603910325 & 1.000031603986271 & $7.59461383 \mathrm{e}-11$ \\
0.500000 & 0.999985898306111 & 0.999985898706800 & $4.00689260 \mathrm{e}-10$ \\
0.562500 & 1.000027707908093 & 1.000027708086553 & $1.78460358 \mathrm{e}-10$ \\
0.625000 & 1.000029576187069 & 1.000029576392916 & $2.05846895 \mathrm{e}-10$ \\
\hline
\end{tabular}

The mean absolute error is $2.219070815989710 \mathrm{e}-010$.

Table 2 above shows the exact solution, numerical solution and absolute error for the effect of using stepsize $2^{-5}$. (See Appendix 2 for the graph of the result on Table 2). 
Table 3. Results of problem 1 showing the effect of using stepsize $2^{-6}$.

\begin{tabular}{llll}
\hline t-value & Exact Solution & Numerical Solution & Absolute Error \\
\hline 0.062500 & 0.999993968111180 & 0.999993968149564 & $3.83839627 \mathrm{e}-11$ \\
0.125000 & 0.999950903398081 & 0.999950903590571 & $1.92489358 \mathrm{e}-10$ \\
0.187500 & 1.000018522444389 & 1.000018522445110 & $7.20534743 \mathrm{e}-13$ \\
0.250000 & 0.999969318836642 & 0.999969319010660 & $1.74018133 \mathrm{e}-10$ \\
0.312500 & 0.999995025525107 & 0.999995025638328 & $1.13220988 \mathrm{e}-10$ \\
0.375000 & 0.999971494315777 & 0.999971494522059 & $2.06282436 \mathrm{e}-10$ \\
0.437500 & 1.000023470014742 & 1.000023470078141 & $6.33995079 \mathrm{e}-11$ \\
0.500000 & 1.000006560271671 & 1.000006560407446 & $1.35774725 \mathrm{e}-10$ \\
0.562500 & 1.000016550151243 & 1.000016550275336 & $1.24092958 \mathrm{e}-10$ \\
0.625000 & 1.000050801848815 & 1.000050801885408 & $3.65927288 \mathrm{e}-11$ \\
\hline
\end{tabular}

The mean absolute error is $1.084975331799853 \mathrm{e}-010$.

Table 3 above shows the exact solution, numerical solution and absolute error for the effect of using stepsize $2^{-6}$. (See Appendix 3 for the graph of the result on Table 3).

Table 4. Results of problem 1 showing the effect of using stepsize $2^{-7}$.

\begin{tabular}{llll}
\hline t-value & Exact Solution & Numerical Solution & Absolute Error \\
\hline 0.062500 & 0.999960105863295 & 0.999960105935395 & $7.21005478 \mathrm{e}-11$ \\
0.125000 & 0.999967950241945 & 0.999967950311558 & $6.96133151 \mathrm{e}-11$ \\
0.187500 & 0.999964311166688 & 0.999964311251752 & $8.50645110 \mathrm{e}-11$ \\
0.250000 & 0.999983928766161 & 0.999983928830341 & $6.41801057 \mathrm{e}-11$ \\
0.312500 & 1.000010033769263 & 1.000010033802423 & $3.31599193 \mathrm{e}-11$ \\
0.375000 & 1.000060677640722 & 1.000060677604517 & $3.62043728 \mathrm{e}-11$ \\
0.437500 & 1.000067288106194 & 1.000067288069427 & $3.67670339 \mathrm{e}-11$ \\
0.500000 & 1.000122399331946 & 1.000122399218831 & $1.13115517 \mathrm{e}-10$ \\
0.562500 & 1.000162783152388 & 1.000162782985934 & $1.66453296 \mathrm{e}-10$ \\
0.625000 & 1.000180536086851 & 1.000180535902423 & $1.84428250 \mathrm{e}-10$ \\
\hline
\end{tabular}

The mean absolute error is $8.610868684755246 \mathrm{e}-011$.

Table 4 above shows the exact solution, numerical solution and absolute error for the effect of using stepsize $2^{-7}$. (See Appendix 4 for the graph of the result on Table 4).

Table 5. Results of problem 1 showing the effect of using stepsize $2^{-8}$.

\begin{tabular}{llll}
\hline t-value & Exact Solution & Numerical Solution & Absolute Error \\
\hline 0.062500 & 0.999972159883026 & 0.999972159909660 & $2.66341393 \mathrm{e}-11$ \\
0.125000 & 0.999978280985938 & 0.999978281012673 & $2.67352807 \mathrm{e}-11$ \\
0.187500 & 1.000027372023912 & 1.000027372017179 & $6.73305856 \mathrm{e}-12$ \\
0.250000 & 1.000027372023912 & 1.000027372017179 & $6.73305856 \mathrm{e}-12$ \\
0.312500 & 1.000101764246374 & 1.000101764191288 & $5.50863799 \mathrm{e}-11$ \\
0.375000 & 1.000108726423491 & 1.000108726367848 & $5.56432678 \mathrm{e}-11$ \\
0.437500 & 1.000148957998176 & 1.000148957915983 & $8.21926971 \mathrm{e}-11$ \\
0.500000 & 1.000172569073211 & 1.000172568977453 & $9.57578461 \mathrm{e}-11$ \\
0.562500 & 1.000206392121796 & 1.000206392004494 & $1.17302168 \mathrm{e}-10$ \\
0.625000 & 1.000193604241676 & 1.000193604139249 & $1.02426956 \mathrm{e}-10$ \\
\hline
\end{tabular}

The mean absolute error is $6.004124974978709 \mathrm{e}-011$.

Table 5 above shows the exact solution, numerical solution and absolute error for the effectof using stepsize $2^{-8}$. (See Appendix 5 for the graph of the result on Table 5).

Table 6. Results of problem 1 showing the effect of using stepsize $2^{-9}$.

\begin{tabular}{llll}
\hline t-value & Exact Solution & Numerical Solution & Absolute Error \\
\hline 0.062500 & 0.999979464841858 & 0.999979464852322 & $1.04635189 \mathrm{e}-11$ \\
0.125000 & 1.000036197200983 & 1.000036197191726 & $9.25615140 \mathrm{e}-12$ \\
0.187500 & 1.000061346273481 & 1.000061346256842 & $1.66393566 \mathrm{e}-11$ \\
0.250000 & 1.000101309205331 & 1.000101309175522 & $2.98090441 \mathrm{e}-11$ \\
0.312500 & 1.000111004081822 & 1.000111004050668 & $3.11544124 \mathrm{e}-11$ \\
0.375000 & 1.000054041052400 & 1.000054041045940 & $6.45949960 \mathrm{e}-12$ \\
0.437500 & 1.000068042560500 & 1.000068042551012 & $9.48752188 \mathrm{e}-12$ \\
0.500000 & 1.000067650976551 & 1.000067650969659 & $6.89204249 \mathrm{e}-12$ \\
0.562500 & 1.000133899286137 & 1.000133899255808 & $3.03292946 \mathrm{e}-11$ \\
0.625000 & 1.000143970608418 & 1.000143970576596 & $3.18221005 \mathrm{e}-11$ \\
\hline
\end{tabular}

The mean absolute error is $1.823129425204684 \mathrm{e}-011$ 
Table 6 above shows the exact solution, numerical solution and absolute error for the effect of using stepsize $2^{-9}$. (See Appendix 6 for the graph of the result on Table 6)

Problem 2

$$
d X(t)=-\left(a+b^{2} X^{2}(t)\right)\left(\left(1-X^{2}(t)\right) d t+b\left(1-X^{2}(t)\right) d W(t)\right.
$$

Where $a=0.0002, b=0.0001, t \in[0,1]$ and $X\left(t_{0}\right)=X_{0}$. The true solution is

$$
X(t)=\frac{\left(1+X_{0}\right) e^{(-2 a t+2 b W(t))}+X_{0}-1}{\left(\left(1+X_{0}\right) e^{(-2 a t+2 b W(t))}-X_{0}+1\right)}
$$

Problem 2 was also used by Fadugba et al (2013) with constant $a=1, b=2$.

The following step sizes shall be used to carry out the investigation, $2^{-4}, 2^{-5}, 2^{-6}, 2^{-7}, 2^{-8}, 2^{-9}$. We will assume here that $a=0.0002, b=0.0001$ and $X(0)=-2$ for better accuracy.

Table 7. Results of problem 2 showing the effect of using stepsize $2^{-4}$.

\begin{tabular}{llll}
\hline t-value & Exact Solution & Numerical Solution & Absolute Error \\
\hline 0.062500 & -2.000030639939910 & -2.000030642409364 & $2.46945397 \mathrm{e}-9$ \\
0.125000 & -1.999826604550993 & -1.999826597757476 & $6.79351708 \mathrm{e}-9$ \\
0.187500 & -1.999771190197802 & -1.999771181571479 & $8.62632321 \mathrm{e}-9$ \\
0.250000 & -1.999738855905664 & -1.999738846600268 & $9.30539579 \mathrm{e}-9$ \\
0.312500 & -1.999549931800135 & -1.999549913990723 & $1.78094122 \mathrm{e}-8$ \\
0.375000 & -1.999485167195670 & -1.999485147086992 & $2.01086778 \mathrm{e}-8$ \\
0.437500 & -1.999441604632085 & -1.999441583283593 & $2.13484912 \mathrm{e}-8$ \\
0.500000 & -1.999255786341376 & -1.999255756647863 & $2.96935132 \mathrm{e}-8$ \\
0.562500 & -1.999277377750062 & -1.999277350071063 & $2.76789982 \mathrm{e}-8$ \\
0.625000 & -1.999295108719981 & -1.999295082862801 & $2.58571793 \mathrm{e}-8$ \\
\hline
\end{tabular}

The mean absolute error is $1.696909619486320 \mathrm{e}-008$.

Table 7 above shows the exact solution, numerical solution and absolute error for the effectof using stepsize $2^{-4}$. (See Appendix 7 for the graph of the result on Table 7).

Table 8. Results of problem 2 showing the effect of using stepsize $2^{-5}$.

\begin{tabular}{llll}
\hline t-value & Exact Solution & Numerical Solution & Absolute Error \\
\hline 0.062500 & -1.999892917380804 & -1.999892915172975 & $2.20782859 \mathrm{e}-9$ \\
0.125000 & -1.999846392834979 & -1.999846389932891 & $2.90208790 \mathrm{e}-9$ \\
0.187500 & -1.999682499484875 & -1.999682492955720 & $6.52915455 \mathrm{e}-9$ \\
0.250000 & -1.999535775997482 & -1.999535766271172 & $9.72630998 \mathrm{e}-9$ \\
0.312500 & -1.999579115423757 & -1.999579107248484 & $8.17527313 \mathrm{e}-9$ \\
0.375000 & -1.999694550513440 & -1.999694545690344 & $4.82309592 \mathrm{e}-9$ \\
0.437500 & -1.999569940134597 & -1.999569932666208 & $7.46838902 \mathrm{e}-9$ \\
0.500000 & -1.999357977043176 & -1.999357964749685 & $1.22934911 \mathrm{e}-8$ \\
0.562500 & -1.999408364465035 & -1.999408353898191 & $1.05668438 \mathrm{e}-8$ \\
0.625000 & -1.999339028019188 & -1.999339016188690 & $1.18304986 \mathrm{e}-8$ \\
\hline
\end{tabular}

The mean absolute error is $7.652297262517039 \mathrm{e}-009$.

Table 8 above shows the exact solution, numerical solution and absolute error for the effectof using stepsize 2-5. (See Appendix 8 for the graph of the result on Table 8).

Table 9. Results of problem 2 showing the effect of using stepsize $2^{-6}$.

\begin{tabular}{llll}
\hline t-value & Exact Solution & Numerical Solution & Absolute Error \\
\hline 0.062500 & -1.999906910993895 & -1.999906910064862 & $9.29032185 \mathrm{e}-10$ \\
0.125000 & -1.999702767364218 & -1.999702764118717 & $3.24550031 \mathrm{e}-9$ \\
0.187500 & -1.999830588766838 & -1.999830587352560 & $1.41427758 \mathrm{e}-9$ \\
0.250000 & -1.999608061287530 & -1.999608057327698 & $3.95983291 \mathrm{e}-9$ \\
0.312500 & -1.999610182559322 & -1.999610178860159 & $3.69916386 \mathrm{e}-9$ \\
0.375000 & -1.999464678473431 & -1.999464673190970 & $5.28246158 \mathrm{e}-9$ \\
0.437500 & -1.999545553506756 & -1.999545549468417 & $4.03833855 \mathrm{e}-9$ \\
0.500000 & -1.999419912678686 & -1.999419907305332 & $5.37335354 \mathrm{e}-9$ \\
0.562500 & -1.999374919082127 & -1.999374913380986 & $5.70114156 \mathrm{e}-9$ \\
0.625000 & -1.999402649038457 & -1.999402643917728 & $5.12072984 \mathrm{e}-9$ \\
\hline
\end{tabular}

The mean absolute error is $3.876383192213950 \mathrm{e}-009$. 
Table 9 above shows the exact solution, numerical solution and absolute error for the effectof using stepsize $2^{-6}$. (See Appendix 9 for the graph of the result on Table 9).

Table 10. Results of problem 2 showing the effect of using stepsize $2^{-7}$.

\begin{tabular}{llll}
\hline t-value & Exact Solution & Numerical Solution & Absolute Error \\
\hline 0.062500 & -1.999805341404871 & -1.999805340305775 & $1.09909570 \mathrm{e}-9$ \\
0.125000 & -1.999753891446814 & -1.999753890143567 & $1.30324640 \mathrm{e}-9$ \\
0.187500 & -1.999668007898771 & -1.999668006176140 & $1.72263093 \mathrm{e}-9$ \\
0.250000 & -1.999651870477482 & -1.999651868771152 & $1.70633041 \mathrm{e}-9$ \\
0.312500 & -1.999655185128882 & -1.999655183560381 & $1.56850133 \mathrm{e}-9$ \\
0.375000 & -1.999732080887753 & -1.999732079916673 & $9.71079883 \mathrm{e}-10$ \\
0.437500 & -1.999676933687320 & -1.999676932488846 & $1.19847443 \mathrm{e}-9$ \\
0.500000 & -1.999767219155966 & -1.999767218638566 & $5.17400789 \mathrm{e}-10$ \\
0.562500 & -1.999813341382202 & -1.999813341270112 & $1.12089893 \mathrm{e}-10$ \\
0.625000 & -1.999791597710221 & -1.999791597579308 & $1.30913502 \mathrm{e}-10$ \\
\hline
\end{tabular}

The mean absolute error is $1.032976326698076 \mathrm{e}-009$.

Table 10 above shows the exact solution, numerical solution and absolute error for the effect of using stepsize $2^{-7}$. (See Appendix 10 for the graph of the result on Table 10).

Table 11. Results of problem 2 showing the effect of using stepsize $2^{-8}$.

\begin{tabular}{llll}
\hline t-value & Exact Solution & Numerical Solution & Absolute Error \\
\hline 0.062500 & -1.999841496174841 & -1.999841495738286 & $4.36554792 \mathrm{e}-10$ \\
0.125000 & -1.999784874982539 & -1.999784874427676 & $5.54862600 \mathrm{e}-10$ \\
0.187500 & -1.999857131369450 & -1.999857131098878 & $2.70572231 \mathrm{e}-10$ \\
0.250000 & -1.999897512575721 & -1.999897512489857 & $8.58642046 \mathrm{e}-11$ \\
0.312500 & -1.999930285134005 & -1.999930285209092 & $7.50870477 \mathrm{e}-11$ \\
0.375000 & -1.999876177386879 & -1.999876177351470 & $3.54092311 \mathrm{e}-11$ \\
0.437500 & -1.999921851349309 & -1.999921851515182 & $1.65873315 \mathrm{e}-10$ \\
0.500000 & -1.999917674573231 & -1.999917674784625 & $2.11394457 \mathrm{e}-10$ \\
0.562500 & -1.999944122996693 & -1.999944123349270 & $3.52577079 \mathrm{e}-10$ \\
0.625000 & -1.999830784974864 & -1.999830785031834 & $5.69697622 \mathrm{e}-11$ \\
\hline
\end{tabular}

The mean absolute error is $2.245164720804382 \mathrm{e}-010$.

Table 11 above shows the exact solution, numerical solution and absolute error for the effectof using stepsize $2^{-8}$. (See Appendix 11 for the graph of the result on Table 11).

Table 12. Results of problem 2 showing the effect of using stepsize $2^{-9}$

\begin{tabular}{llll}
\hline t-value & Exact Solution & Numerical Solution & Absolute Error \\
\hline 0.062500 & -1.999863407270136 & -1.999863407086074 & $1.84061877 \mathrm{e}-10$ \\
0.125000 & -1.999958592655718 & -1.999958592649633 & $6.08535444 \mathrm{e}-12$ \\
0.187500 & -1.999959037106798 & -1.999959037130689 & $2.38906672 \mathrm{e}-11$ \\
0.250000 & -2.000003915981924 & -2.000003916105223 & $1.23299593 \mathrm{e}-10$ \\
0.312500 & -1.999957999627532 & -1.999957999708359 & $8.08273448 \mathrm{e}-11$ \\
0.375000 & -1.999712179640226 & -1.999712179366374 & $2.73852718 \mathrm{e}-10$ \\
0.437500 & -1.999679195925699 & -1.999679195629594 & $2.96105140 \mathrm{e}-10$ \\
0.500000 & -1.999603058636733 & -1.999603058251007 & $3.85726118 \mathrm{e}-10$ \\
0.562500 & -1.999726729199231 & -1.999726729035875 & $1.63356217 \mathrm{e}-10$ \\
0.625000 & -1.999681957561421 & -1.999681957357425 & $2.03995709 \mathrm{e}-10$ \\
\hline
\end{tabular}

The mean absolute error is $1.741200739147075 \mathrm{e}-010$.

Table 12 above shows the exact solution, numerical solution and absolute error for the effect of using stepsize $2^{-9}$. (See Appendix 12 for the graph of the result on Table 12).

\section{Determination of Order of Convergence of the Method}

To determine the accuracy of any numerical method, the properties of methods of solution of stochastic differential equations cannot be ignored. Some of the properties peculiar 
to SDEs include convergence and order of convergence. The issues of convergence of SDEs have been examine by Higham (2001), Burrage (2004), Lactus (2008), Sauer (2013), Fadugba et al (2013) and Akinbo et al (2015) for one step method etc.

In this section, mean absolute error (MAE) or Strong Error of one step MLSTM will be determined to assess the effect of varying stepsizes. The order of convergence of the method will be obtained using mean absolute error (MAE) obtained.

Table 13(a) and (b): Results showing MAE of one step MLSTM applied to problems 1 and 2 with varying stepsizes.

Table 13(a). MAE for Problem 1.

\begin{tabular}{ll}
\hline Stepsize & Mean Absolute error \\
\hline $2-4$ & $5.87318316 \mathrm{e}-10$ \\
$2-5$ & $2.21907082 \mathrm{e}-10$ \\
$2-6$ & $1.08497533 \mathrm{e}-10$ \\
$2-7$ & $8.61086868 \mathrm{e}-11$ \\
$2-8$ & $6.00412497 \mathrm{e}-11$ \\
$2-9$ & $1.82312943 \mathrm{e}-11$ \\
\hline
\end{tabular}

Table 13(b). MAE for Problem 2.

\begin{tabular}{ll}
\hline Stepsize & Mean Absolute error \\
\hline $2-4$ & $1.69690962 \mathrm{e}-8$ \\
$2-5$ & $7.65229726 \mathrm{e}-9$ \\
$2-6$ & $3.87638319 \mathrm{e}-9$ \\
$2-7$ & $1.03297633 \mathrm{e}-9$ \\
$2-8$ & $2.24516472 \mathrm{e}-10$ \\
$2-9$ & $1.74120074 \mathrm{e}-10$ \\
\hline
\end{tabular}

Using the data in table 13a, the strong order of convergence $\lambda$ for one step MLSTM and the residual $C$ for one step MLSTM with respect to problem 1 can be obtained by making least squares fit using MALAB commands. Running the MATLAB commands, the strong convergence of order $\lambda \simeq 0.8868$ while the residual $C \simeq 0.5739$. Similarly, for problem 2 , the strong convergence of order $\lambda \simeq 1.4347$ while the residual $C \simeq 0.6808$.

\section{Discussion}

We have so far discussed about the method of deriving one step method of Milstein type. This method was applied to two SDEs. The method was used to determine the numerical solution of the given problem. Absolute errors were calculated using the numerical approximation and the corresponding exact solution. Mean absolute error were determined. Using this, comparison of absolute error was made. To determine the accuracy of the method, strong order of convergence was obtained for each method. The order of convergence obtained was approximately 1 which shows the accuracy of the method.

\section{Conclusion}

In this paper, two problems in the form of first order SDEs have been considered.One step Milstein method (MLSTM) for solution of general first order stochastic differential equations (SDEs) has been derived. The absolute errors between the exact solution and numerical solution have been determined using this method. The mean absolute errors for varying stepsizes were also determined. The result showed that the mean absolute error generally decreases as the stepsizes decreases. The effect of the varying stepsizes can also be seen by observing the behaviour of the exact solution and numerical solution using graphical method as indicated in Appendix 1 to 12. To determine the accuracy of the method, we also determine the strong order of convergence of the method. For problem 1 the strong order of convergence gives $\lambda \simeq 0.8868$ while for problem 2 , the strong order of convergence gives $\lambda \simeq 1.4347$. The results were obtained using MATLAB as a supporting tool.

\section{Appendix 1}

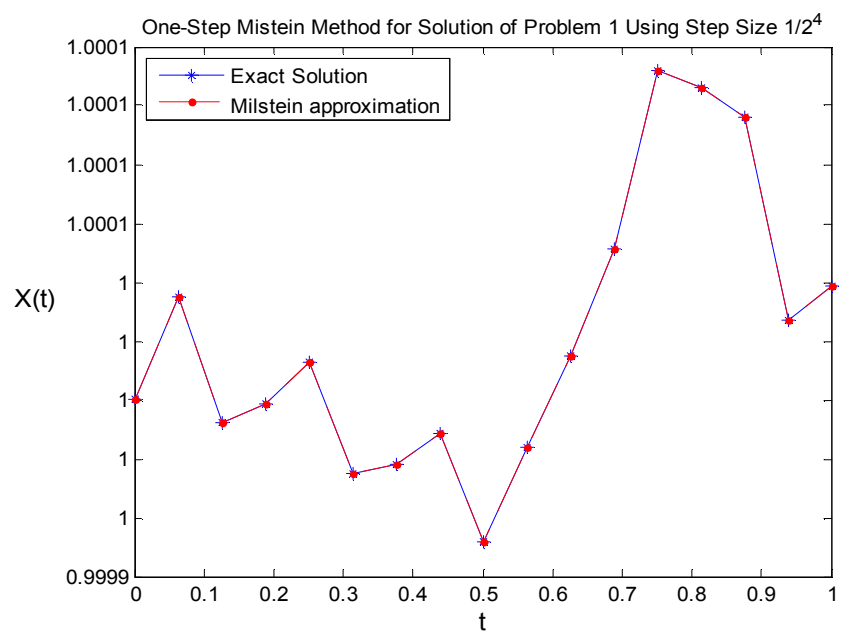

Figure 1. Shows the graph of exact solution and one step Milstein Method with stepsize $2^{-4}$.

\section{Appendix 2}

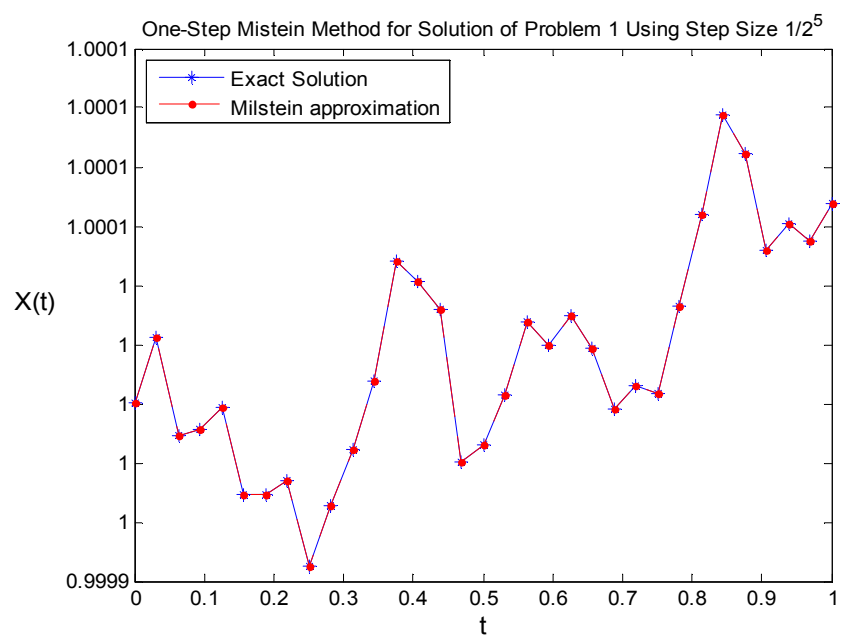

Figure 2. Shows the graph of exact solution and one step Milstein Method with stepsize $2^{-5}$. 


\section{Appendix 3}

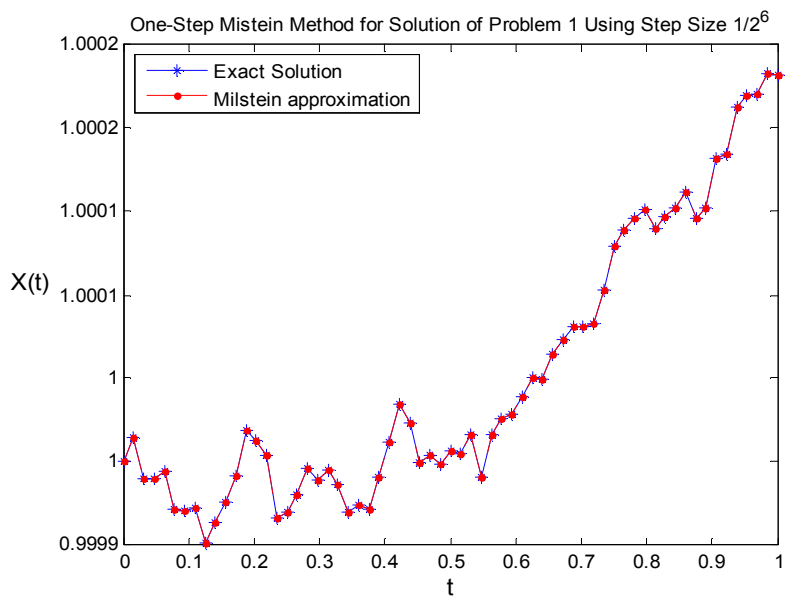

Figure 3. Shows the graph of exact solution and one step Milstein Method with stepsize $2^{-6}$.

\section{Appendix 4}

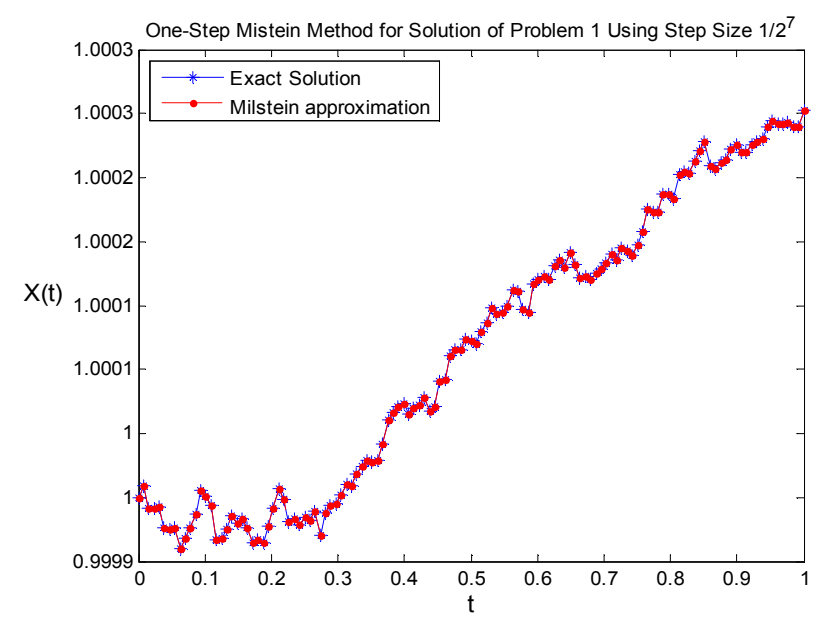

Figure 4. Shows the graph of exact solution and one step Milstein Method with stepsize $2^{-7}$.

\section{Appendix 5}

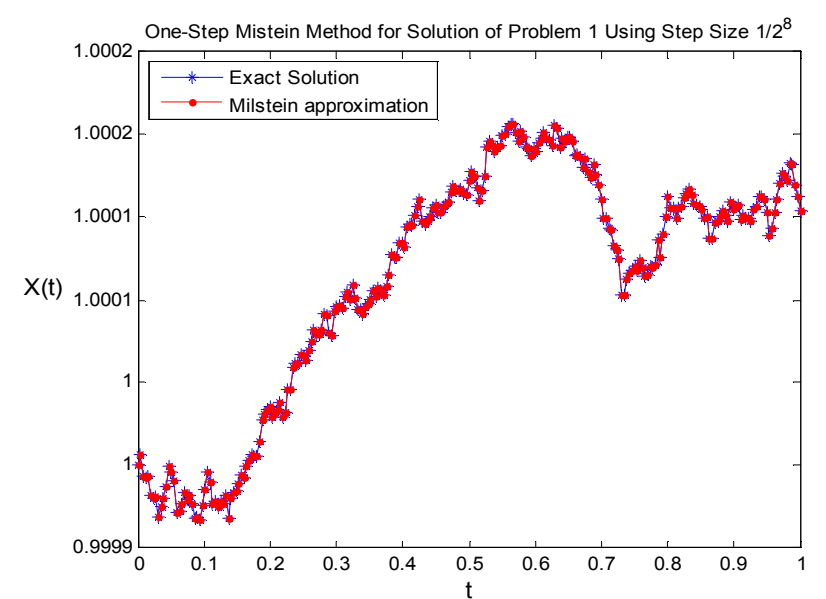

Figure 5. Shows the graph of exact solution and one step Milstein Method with stepsizes $2^{-8}$.

\section{Appendix 6}

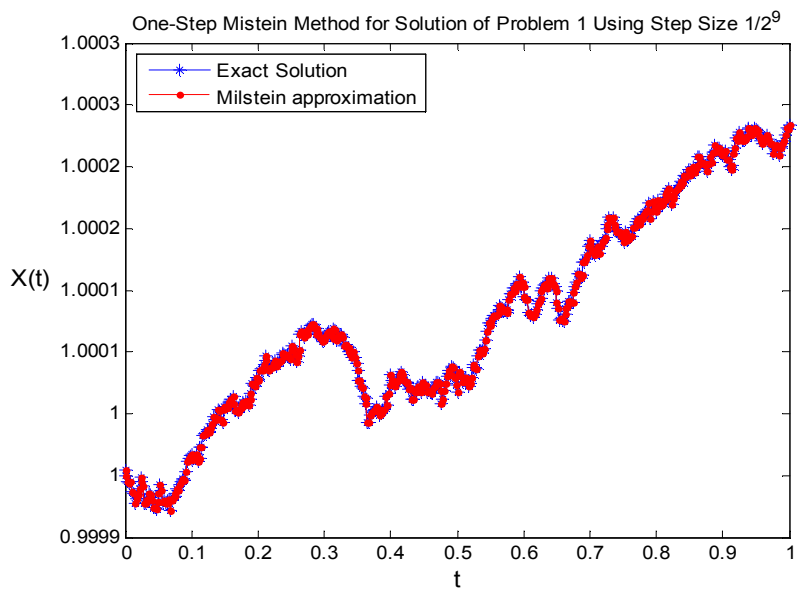

Figure 6. Shows the graph of exact solution and one step Milstein Method with stepsizes $2^{-9}$.

\section{Appendix 7}

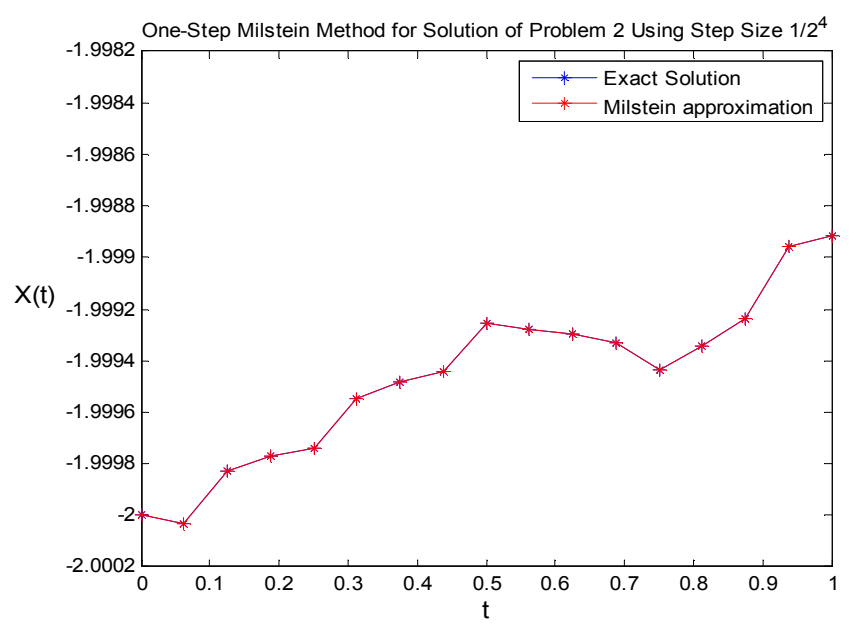

Figure 7. Shows the graph of exact solution and one step Milstein Method with stepsizes $2^{-4}$.

\section{Appendix 8}

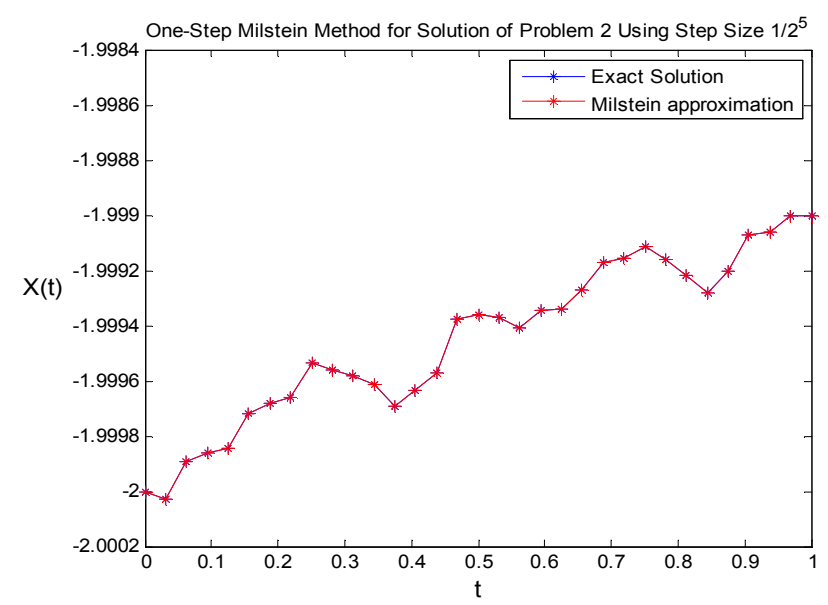

Figure 8. Shows the graph of exact solution and one step Milstein Method with stepsizes $2^{-5}$. 


\section{Appendix 9}

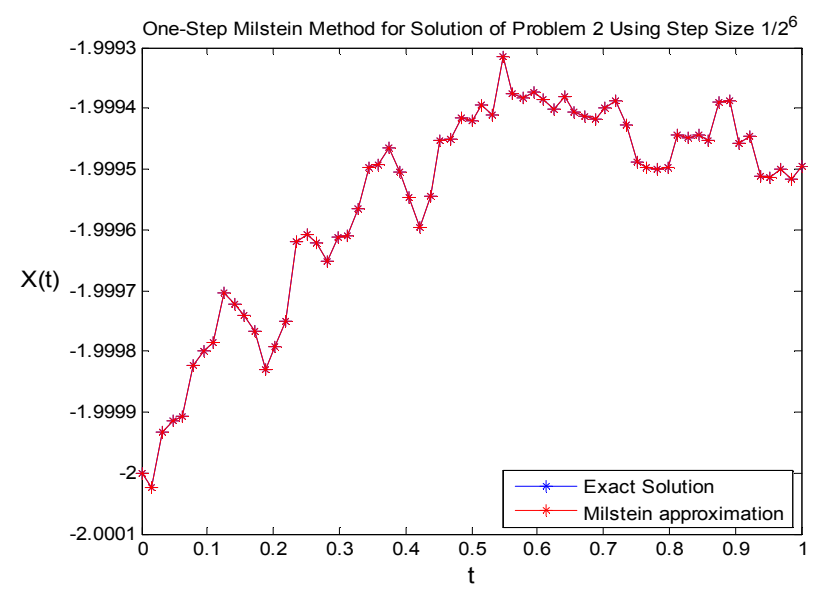

Figure 9. Shows the graph of exact solution and one step Milstein Method with stepsizes $2^{-6}$.

\section{Appendix 10}

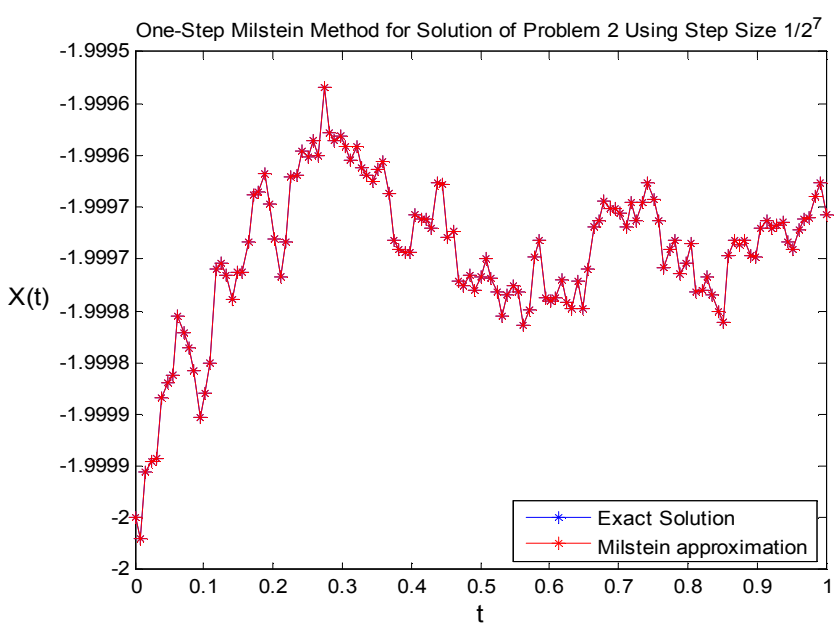

Figure 10. Shows the graph of exact solution and one step Milstein Method with stepsizes $2^{-7}$.

\section{Appendix 11}

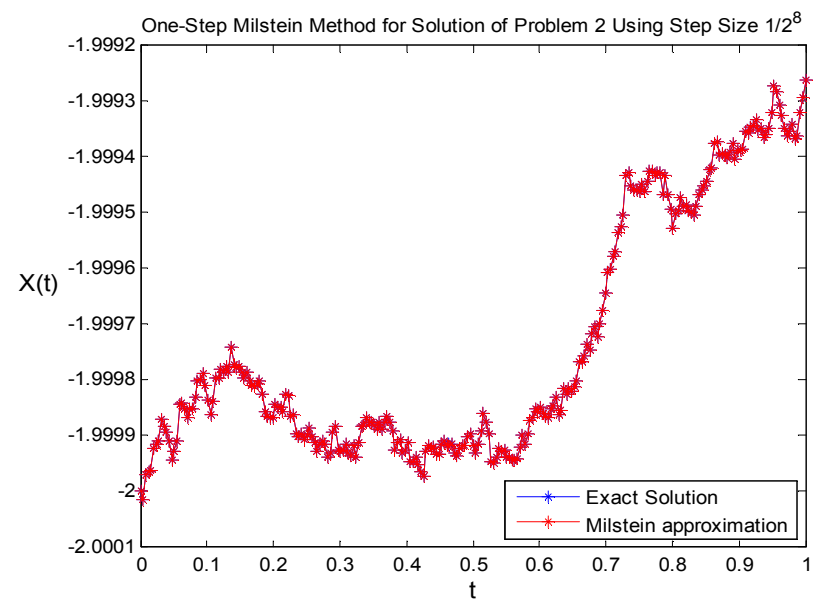

Figure 11. Shows the graph of exact solution and one step Milstein Method with stepsizes $2^{-8}$.

\section{Appendix 12}

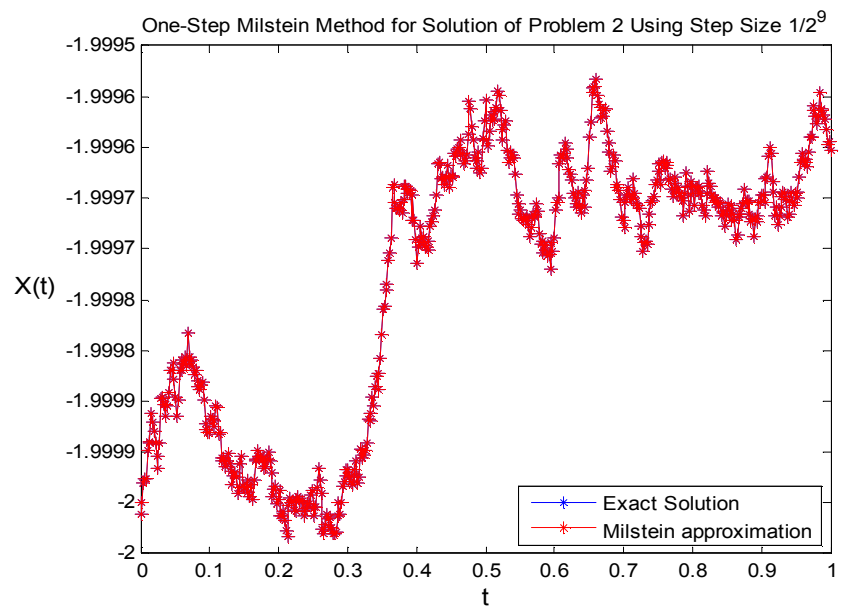

Figure 12. Shows the graph of exact solution and one step Milstein Method with stepsizes $2^{-9}$.

\section{References}

[1] Akinbo B.J., Faniran T. and Ayoola E.O. (2015). Numerical Solution of Stochastic Differential Equations. International Journal of Advanced Research in Science, Engineering and Technology.

[2] Anna N. (2010). Economical Runge-Kutta Methods with week Second Order for Stochastic Differential Equations. Int. Contemp. Maths. Sciences, 5(24), 24 1151-1160.

[3] Beretta, M., Carletti, F. and Solimano F. (2000). "On the Effects of Enviromental Flunctuationsin Simple Model of BscteriaBacteriophage Interaction, Canad. Appl. Maths. Quart. 8(4)321-366.

[4] Bokor R.H. (2003). "Stochastically Stable One Step Approximations of Solutions of Stochastic Ordinary Differential Equations", J. Applied Numerical Mathematics 44, 21-39.

[5] Burrage, K. (2004). Numerical Methods for Strong Solutions of Stochastic Differential Equations: An overview. Proceedings: Mathematical Physical and Engineering Science, Published by Royal Society, 460(2041), 373-402.

[6] Burrage, K. Burrage, P. and Mitsui T. (2000). Numerical Solutions of Stochastic Differential EquationsImplementation and Stability Issues. Journalof Computational\&Applied Mathematics, 125, 171-182.

[7] Fadugba S.E., Adegboyegun B.J., and Ogunbiyi O.T. (2013). On Convergence of Euler-Maruyama and Milstein Scheme for Solution of Stochastic Differential Equations. International Journal of Applied Mathematics and Modeling@ KINDI PUBLICATIONS.1(1), 9-15. ISSN: 2336-0054.

[8] Higham D.J. (2001). An Algorithmic Introduction to Numerical Simulation of Stochastic Differential Equations. SIAM Review, 43(3), 525-546.

[9] Lactus, M.L. (2008). Simulation and Inference for Stochastic Differential Equations withR Examples. Springer Science + Buisiness Media, LLC, 233 Springer Street, New York,NY10013, USA. Pp 61-62. 
[10] Oksendal B. (1998). Stochastic Differential Equations. An Introduction with Application, FifthEdition, Springer-Verlage, Berlin, Heidelberge, Italy.

[11] Platen E. (1992). An Introduction to Numerical Methods of Stochastic Differential equations. ActaNumerica, 8, 197-246.

[12] Rezaeyan R. and Farnoosh R. (2010). Stochastic Differential Equations and Application of Kalman-Bucy Filter in Modeling of RC Circuit. Applied Mathematical Sciences, Stochastic Differential Equations 4(33), 1119-1127.

[13] Richardson M. (2009). Stochastic Differential Equations Case
Study. (Unpublished).Sauer T. (2013). Computational Solution of Stochastic Differential Equations. WIRES ComputStat. doi: 10.1002/wics.1272.

[14] Wang P. and Liu Z. (2009). "Stabilized Milstein Type Methods for Stiff Stochastic Systems". Journal of Numerical Mathematics and Stochastics, Eulidean Press, LLC. 1(1): 3334.

[15] Yashihiro S. and Taketomo M. (1996). Stability Analysis of Numerical Schemes for Stochastic Differential Equations. SIAM J. Numer. Anal. 33(6), 2254. 